\title{
Gargantuanperipheral Cemento-Ossifying Fibroma- A Case Report
}

\author{
Dr Kiran Kumar $\mathrm{N}^{1}$, Dr Dandena Vinay Kumar ${ }^{2}$, Dr Manjunath Reddy ${ }^{3}$ \\ Dr Pradeep M R ${ }^{4}$. \\ ${ }^{I}$ (Conservative Dentistry and Endodontics, Gdcri Bangalore/Rguhs, India) \\ 2,4 (Oral Pathology,Ame's Dental College And Hospital Raichur/Rguhs,India) \\ ${ }_{3}^{3}$ (Oral Surgery Gdc Ballari/Rguhs India)
}

\begin{abstract}
Cemento-ossifying fibroma (COF) is a benign neoplasm characterized by replacement of normal bone by fibrous tissue and varying amounts of newly formed bone or cementum-like material, or both. ${ }^{l}$ Peripheral Cemento ossifying fibroma (PCOF) is a reactive lesion which has been described with various synonyms and is believed to arise from the periodontal ligament. ${ }^{2}$ It predominantly affects adolescents and young adults, with peak prevalence between 10 and 19 yrs. There is a definite female predilection and almost $60 \%$ of the lesions occur in the maxilla. Trauma or local irritation such as dental calculus, ill-fitting denture appliances and faulty restorations are known to precipitate the development of this lesion. ${ }^{3}$ The present report describes a case of Peripheral Cemento ossifying fibroma (PCOF) in a 60 year old female with a mass on anterior labial and palatal gingival region.
\end{abstract}

Keywords: $\mathrm{COF}, \mathrm{PCOF}$

\section{Introduction}

Ossifying fibroma is a neoplasm composed of fibrous tissue that contains a variable mixture of bony trabeculae, cementum like spherules or both. There are two types of ossifying fibroma, central and peripheral. The central type arises from the endosteum or periodontal ligament adjacent to the root apex and expands from the medullary cavity of the bone whereas the peripheral type occurs solely on the soft tissue overlying the alveolar process and is a 'non-neoplastic', reactive fibrous proliferation of gingiva. ${ }^{4}$ Many of the fibrous growths originate from underneath the periodontium as similar to peripheral Cemento-ossifying fibroma (PCOF). PCOF is an example of such an occasionally arising growth on the gingiva. Peripheral Cementoossifying fibroma (PCOF) accounts for 3.1\% of all oral tumors and for $9.6 \%$ of gingival lesions. PCOF affects both genders but a higher predilection for females has been reported. ${ }^{5}$

Case report: A 60 year old female patient presented with complaint of painless swelling in anterior maxillary region since six months. She is known diabetic and under medication, general physical status of the patient is good. On extra oral examination growth is visible and lips are potentially incompetent (Figure1). Lymph nodes are not palpable. On intra oral examination Swelling is hard, erythematous with few ulcerated areas extends from the labial vestibule to the posterior palatal region bilaterally and it is around $4 \times 5 \mathrm{~cm}$ in size. (Figure 2) Oral hygiene is poor as she can't brush properly due to the presence of growth. On radiographic examination the lateral cephalogram shows specks of radio opacity in the anterior region.(Figure 3)Aprovisional diagnosis of ossifying fibroma is given. Incisional biopsy was done under LA and sent to oral pathology department for histopathological examination. On microscopic examination diagnosis of peripheral Cemento ossifying fibroma was given. Patient underwent surgery and excised the entire growth under GA. On Gross examination it measures around $4.5 \times 4 \times 4 \mathrm{~cm}$, oval in shape, firm in consistency, whitish in color. Multiple bits sent for processing. (Figure 4)

On microscopic examination it shows parakeratinised stratified squamous epithelium with elongated rete ridges and epithelium is discontinuous in few areas. Underlying connective tissue shows increased vascularity, numerous budding vessels, bundles of collagen fibers and deeper connective tissue shows areas of ossification showing various forms like trabecular pattern, globular (cementum like) and irregular in few areas in a delicate fibrous stroma. Based on clinical, radiograph and histopathology a final diagnosis of peripheral Cemento-ossifying fibroma was made. (Figure $\mathbf{5 a}, \mathbf{5 b}, \mathbf{5 c}$ ) 

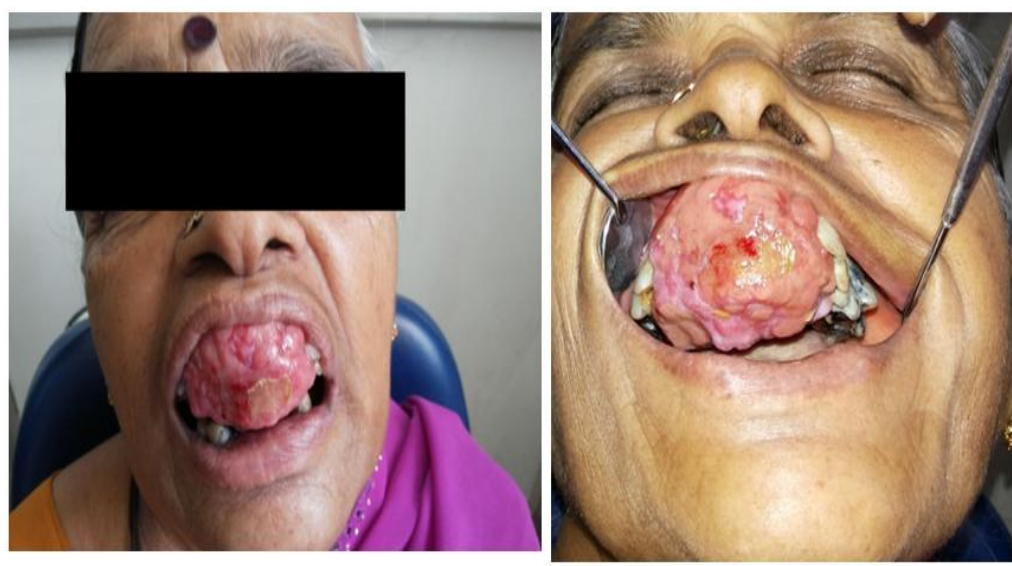

Figure 1 and 2: Extraoral and Intraoral views respectively showing growth in the anterior region extending posteriorly.

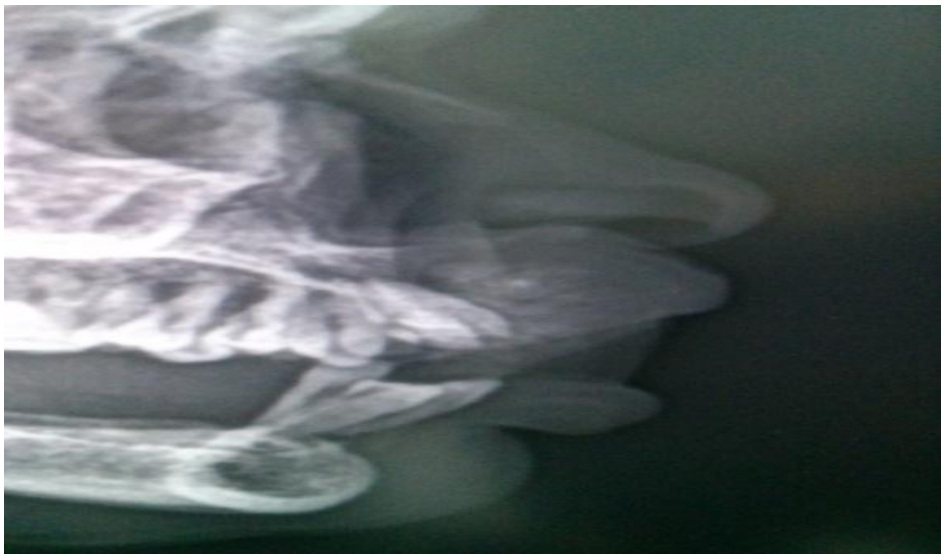

Figure 3: Lateral cephalogram showing specks of radioopacity in the anterior region.

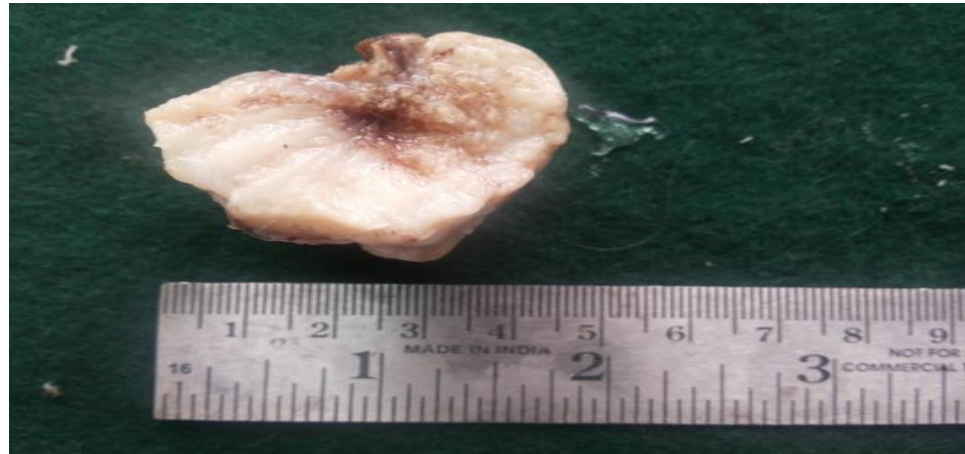

Figure 4: Macroscopy shows whitish with reddish brown surfaces in few areas which measures around $4.5 \times 4 \times 4$ $\mathrm{cm}$

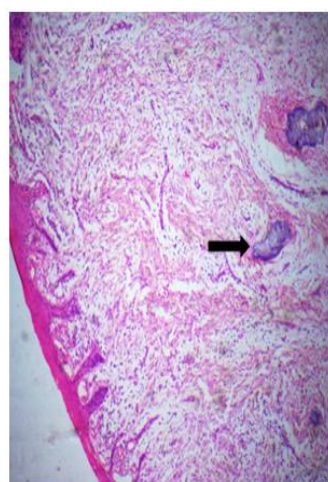

$5 \mathrm{a}$

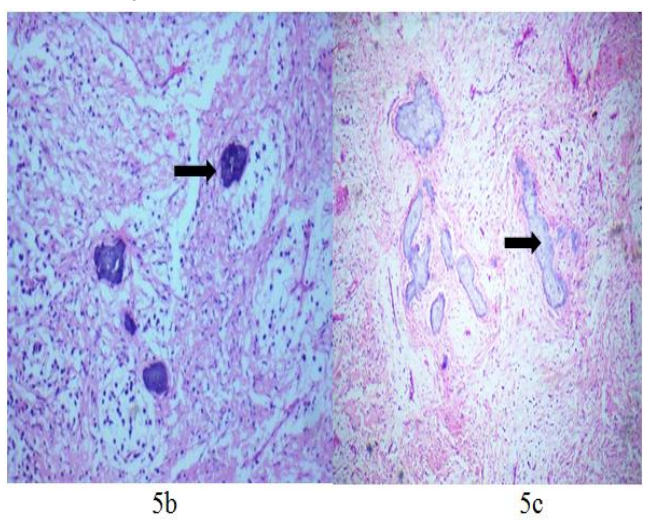

$5 b$ 
Figure 5 (a): $\mathrm{H}$ and $\mathrm{E}$ (40x) showing parakeratinised stratified squamous epithelium and connective tissue showing few globules of cementum like structures. (b): H and E (200x) calcifications (cementum like) in the connective tissue. (c): $\mathrm{H}$ and $\mathrm{E}(200 \mathrm{x})$ calcifications (both cementum like and trabecular) in the connective tissue.

\section{Discussion}

Peripheral Cemento-ossifying fibroma (PCOF) was first described by Menzel in 1827. It has been given many synonyms, such as epulis, calcifying fibroblastic granuloma, peripheral cementifying fibroma, peripheral fibroma with cementogenesis, ossifying fibro epithelial polyp and peripheral fibroma with osteogenesis. Bhasker et al in 1984 described this lesion as peripheral fibroma with calcification and the term PCOF was coined by Eversole and Robin. ${ }^{4}$ Approximately $60 \%$ of PCOFs occur in the maxilla and they are found more often in the anterior region, with $55-60 \%$ presenting in the incisor-cuspid region. ${ }^{3}$

The etiopathogenesis of PCOF is unclear, trauma or local irritants such as subgingival plaque and calculus, dental appliances, poor-quality dental restorations, microorganism, masticatory forces, food lodgment. ${ }^{4}$ PCOFs are believed to arise from gingival fibres of the periodontal ligament as hyperplastic growth of tissue that is unique to the gingival mucosa. This hypothesis is based on the fact that POFs arise exclusively on the gingiva, the subsequent proximity of the gingiva to the periodontal ligament and the inverse correlation between age distribution of patients presenting with POF and the number of missing teeth with associated periodontal ligament. Histopathologically, PCOF can exhibit either an intact or ulcerated stratified squamous epithelium. The deeper fibroblastic component is highly cellular with central areas of calcification. The mineralized tissue may consist of bone, cementum like material, dystrophic calcification, or a combination of each. ${ }^{4}$

Treatment of PCOF consists of elimination of etiological factors, scaling of adjacent teeth and total aggressive surgical excision along with involved periodontal ligament and periosteum to minimize the possibility of recurrence.Our case is that of giant one and etiology may due to stains and calculus (poor oral hygiene) or trauma. Radiographically it shows specks of radio opacity in the anterior region in lateral cephalogram, histopathology shows ulcerated stratified squamous epithelium with mineralized tissue (trabecular bone and cementum like material), which is completely excised under general anaesthesia.

\section{Conclusion}

Close follow up is required because of $8 \%-20 \%$ recurrence rate as it has a growth potential if it is incompletely removed.PCOF clinically resembles as pyogenic granuloma, peripheral giant cell granuloma, so radiographic and histopathological examination is essential for accurate diagnosis.

\section{References}

[1]. David B. Kamadjaja. Cemento-ossifying fibroma of the jaw- A case report. Dental Journal 2009; 40(4):164-71.

[2]. AshishY, Mishra MB. Peripheral cemento ossifying fibroma of mandible a case report. Indian J Stomatol 2011; 2(3):193-96.

[3]. Jaya M, Deepa D, Gopal S, Bhagyashri P. Indian Journal of Basic \& Applied Medical Research. 2013;2(8):1099-1102.

[4]. Priyanka V, Alka W, Savitha B, Hiroj B. Peripheral Cemento Ossifying Fibroma - Case Report. Int J Dent Case Reports 2012; 2(5): $15-18$.

[5]. Suhas G, Sonia G, Girish G, Sandeep G. Cementifying Fibroma of the Mandible - A Case Report. J Oral Health Comm Dent 2008; 2(2):42-45.

[6]. Terry F; Jennifer M, Heather D, Ross. A Peripheral Ossifying Fibroma: A Case Report. JCDA • Www.Cda-Adc.Ca/Jcda 2008; 74(9):809-12. 